\title{
Optimal characteristics of spermatozoa for semen technologies in pigs
}

\author{
I. Parrilla', J.M. Vazquez', I. Caballero'2, M.A. Gil', M. Hernandez', \\ J. Roca', X. Lucas and E.A. Martinez \\ 'Department of Animal Medicine and Surgery, Faculty of Veterinary Medicine, University of Murcia, \\ E-30071, Murcia, Spain. ${ }^{2}$ Department of Molecular Biomedical Sciences, College of Veterinary \\ Medicine, North Carolina State University, Raleigh, NC 27606, USA
}

\begin{abstract}
Despite the great potential of sperm technologies such as sperm cryopreservation and sperm sex sorting for the improvement of different aspects of swine production, artificial insemination with fresh or stored semen is currently the only sperm technology used at a commercial scale in the pig industry. The lower reproductive performance associated with the use of these sperm technologies is the reason for such limited use. Since optimal characteristics are required for successful application of frozenthawed and sex-sorted boar spermatozoa, the present paper summarises the value of the current available methods for their functional assessment as well as the effects of these technologies on boar sperm functionality. In addition, strategies developed to reduce sperm damage and improve the yields of both sperm technologies in swine production are also reviewed with particular attention to the contributions of the authors.
\end{abstract}

\section{Introduction}

Over the past decade, considerable interest has been directed toward the development and improvement of assisted reproductive technologies (ARTs) for swine. Although some ARTs, such as embryo transfer, in vitro production of embryos, cloning and transgenic technology, have been successfully attempted, the current low level of efficiency limits their use in applied production systems. However, ARTs in which only spermatozoa are handled may be applicable for the pig industry in a short period of time (Martinez et al. 2005). Certain sperm technologies, such as cryopreservation of male gametes and sperm sorting for gender pre-selection, might be of great benefit to the swine industry for improving the efficiency of production. Nevertheless, the low reproductive performance associated with the application of these technologies limits their current use.

Many factors come into play in determining the success and spread of sperm technologies. One of these factors is the fertilising ability of the spermatozoa. Handling spermatozoa during technical procedures is usually associated with a decrease in fertilising ability.

Mammalian fertilisation is a complex process involving a precisely programmed set of events during which the spermatozoa acquire their fertilising ability through a series of molecular and cellular changes that are pre-requisite for successful interaction with a female gamete (Petrunkina et al. 2005, Waberski et al. 2005). To be able to carry out these tasks, the spermatozoa must maintain many physical and biochemical parameters that allow it to bind to the zona pellucida and penetrate into the oocyte cytoplasm. In addition, the spermatozoon must have an intact nucleus capable of proper de-condensation, nuclear reorganisation and genetic performance 
in order to maintain proper zygotic and embryonic development (Graham \& Moce 2005). Spermatozoa are cells with a limited biosynthetic capability (Amann et al. 1993), and thus their function can be influenced dramatically by the external environment. Long periods of storage, cryopreservation or sperm sorting by flow cytometry can have a detrimental impact on its function and fertilising ability (Maxwell et al. 1998, Maxwell \& Johnson 1999, Martinez et al. 2001, 2005, Roca et al. 2004, 2006, de Graaf et al. 2008).

In many cases, if not all, the ultimate objective for the practical application of sperm technologies is to obtain optimal reproductive performance. For this reason, special attention should be given to improve the functionality and fertilising ability of the treated spermatozoa.

Since optimal characteristics are required for successful application of frozen-thawed and sex-sorted boar spermatozoa, the present paper summarises the value of the current available methods for their functional assessment as well as the effects of these technologies on boar sperm functionality. In addition, strategies developed to reduce sperm damage and improve the yields of both sperm technologies in swine production are also reviewed with particular attention to the contributions of the authors.

\section{Current procedures to evaluate optimal characteristics of boar spermatozoa}

Sperm technologies such as cryopreservation or flow cytometric sex sorting require extensive in vitro handling, which eventually induces dramatic changes in sperm survivability. Extension, cooling, laser illumination, freezing and thawing, among others, not only cause cell death but also subtly damage most of the spermatozoa in the surviving population, leading to reduced sperm lifespan both in vitro and in vivo (Maxwell et al. 1998). These procedures involve different degrees of change in sperm function following physical and biochemical damage to sperm membranes, DNA, cellular signalling mechanisms, and later on are manifest in alterations to the fertilising capacity of these cells.

Spermatozoa in an ejaculate vary in the integrity of the attributes needed for successful fertilisation as well as in their capability to overcome all the events leading up to fertilisation (Rodriguez-Martinez 2007). Similarly, spermatozoa subjected to technologies such as cryopreservation or sex sorting respond differently to the treatment involved in these procedures. Such variation among cells within a given ejaculate complicates the use of laboratory evaluations as accurate estimation tools (Holt \& Van Look 2004). Moreover, it may be assumed that standard seminal parameters like motility, morphology, and sperm concentration are insufficient to indicate subtle changes in sperm cells subjected to these treatments. Detection of such changes is currently an issue of great importance (Peña et al. 2007, Petrunkina et al. 2007). Therefore, functional assessment analysis should be used to determine minimal and early physical and biochemical damage along with failed metabolic activity in treated sperm samples.

These functional tests include sperm kinematics (assessed by computer-assisted analysis), evaluation of early changes in sperm membranes, evaluation of damage to the sperm chromatin structure, and DNA integrity and sperm-oocyte or sperm-oviduct interactions (Holt et al. 2007, Silva \& Gadella 2006, Petrunkina et al. 2007).

Traditionally, computer-assisted sperm analysis (CASA) systems have been introduced for routine motility evaluations at the commercial level due to their ability to objectively identify sperm motility and motions parameters (Didion 2008). However, in common practice, the use of the mean values of motility descriptors simplifies the motility analysis but does not take into account internal variability, thus providing misleading information about sperm quality, which is not revealed by the usual statistical measures (Holt et al. 2007). New approaches to the 
analysis of data provided by CASA systems involve the identification of sperm subpopulations by means of clustering algorithms. Consequently, these systems offer new insight into the true structure of sperm populations under different experimental conditions and therefore on the relationship between motility, sperm quality and, ultimately, fertility (Cremades et al. 2005; Holt et al. 2007. See Fig. 1). Recently, by means of CASA systems, it has been possible to find computerised systems to objectively estimate sperm head dimensions with a similar statistical approach. Computer-assisted sperm morphometry analysis (CASMA) can be used to distribute spermatozoa in morphometrically distinct subpopulations within the ejaculate and to disclose their relationship with sperm quality (Saravia et al. 2007).

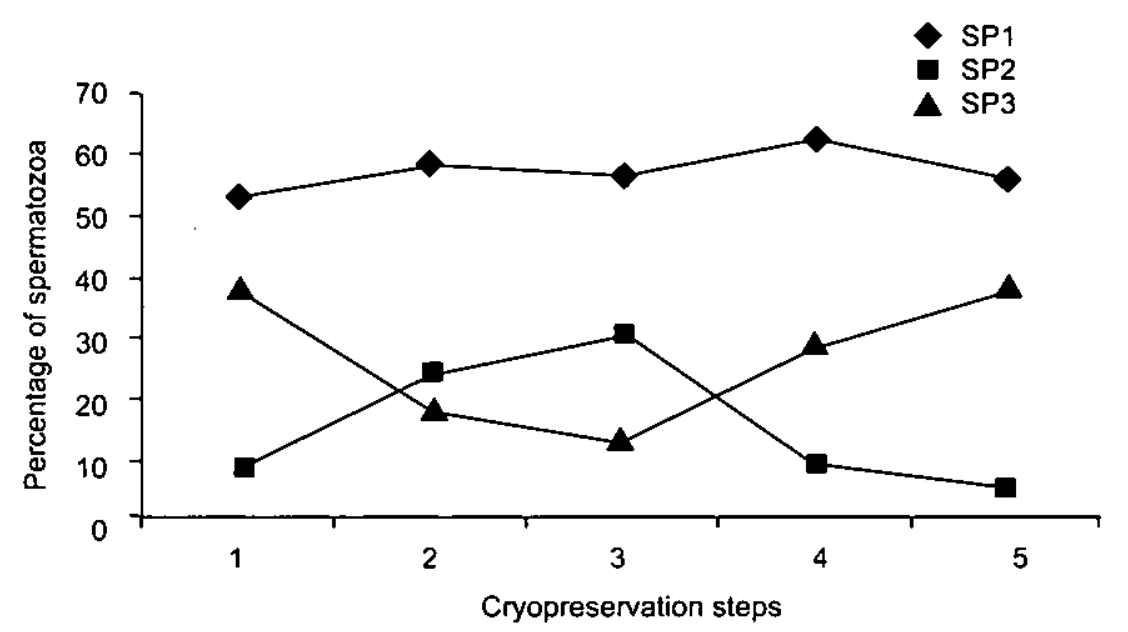

Fig. 1 Behaviour of different motile sperm populations (SP1: cells with progressive and vigorous movement; SP2: progressive cells only; SP3: vigorous cells only, hyperactivelike) identified within one ejaculate by using CASA and statistical analysis during a cryopreservation procedure. The graphic shows how only those spermatozoa belonging to SP1 are able to maintain optimal motility levels during the freezing and thawing processes. The percentages of motile spermatozoa were recorded at five steps during a conventional boar semen cryo-preservation procedure: 1: Immediately after arrival of the BTS-extended sperm-rich ejaculate fraction $\left(22^{\circ} \mathrm{C}\right)$ at the Laboratory of Andrology. 2: After centrifugation and extension of the sperm pellet with LEY extender at $17^{\circ} \mathrm{C}$. 3: After adding the LEYCO extender at $5^{\circ} \mathrm{C}$. 4: After thawing and re-extension with BTS $(1: 1, \mathrm{vol} / \mathrm{vol})$ and holding in a water-bath at $37^{\circ} \mathrm{C}$ for 30 minutes. 5: After holding the re-extended thawed semen in a water bath at $37^{\circ} \mathrm{C}$ for 150 minutes (modified from Cremades et al., 2005).

During recent years, special attention has been paid to plasma membrane integrity since spermatozoa with disrupted plasma membranes are considered unable to fertilise. Plasma membrane integrity is traditionally assessed after staining cells with membrane-permeable dyes such as SYBR-14, combined with propidium iodide as a counter-stain (Silva \& Gadella 2006). Although checking sperm viability is a widespread and highly useful technique for primary sperm quality screening, it cannot discern sub-lethal changes in the sperm cells.

Male gamete structures are extremely sensitive to technological procedures. During these processes, they can suffer a series of events similar to those taking place during sperm capacitation, or as more recently hypothesised, premature ageing or so-called apoptosis-like changes (Peña et al. 2009). In either case, spermatozoa affected by these destabilising events 
undergo membrane changes that apparently lead to cell death (Peña et al. 2009). Some of these changes are linked to early membrane destabilisation and can be evaluated by determining the degree of lipid packing disorder with the lipid dye merocyanine-540 (Harrison \& Gadella 2005 , Guthrie \& Welch 2005) or by investigating membrane asymmetry through changes in the position of certain phospholipids (using Annexin-V) such as phosphatidylserine, which may be trans-located from the inner to the outer leaflet of the plasma membrane after being disturbed (Peña et al. 2003). Changes in membrane permeability imply destabilisation of membranes and can be monitored using the impermeant nuclear dye YO-PRO-1. YO-PRO-1 is used to detect early changes in membrane permeability, alone or in combination with other stains (Peña et al. 2005).

Cell volume is another informative method for the detection of functional membrane changes in live cell populations. The volume regulation capability of spermatozoa is of considerable importance for sperm survival during several processes, including cryopreservation (Petrunkina et al .2005, 2007).

Mitochondrial integrity and functionality are clearly related to sperm viability and motility, and they also play an important role in early sperm changes during technological treatments (Peña et al. 2009). Several dyes have been proposed to evaluate the functional status of these organelles, mostly measuring mitochondrial inner membrane potential, such as JC-1, Mitotracker Green or Mitotracker Deep Red (Guthrie \& Welch 2006, Bussalleu et al. 2005, Hallap et al. 2005).

Many other analyses can be performed to investigate sperm functionality, such as measuring the intracellular calcium concentration (Caballero et al. 2009), stimulating protein tyrosine phosphorylation (Piehler et al. 2006), or searching for the presence of activated caspases (Moran et al. 2008), intracellular reactive oxygen species (Guthrie \& Welch 2006) or other signalling pathways, all of them intimately related to detrimental changes in sperm functionality.

Several studies have shown that spermatozoa with normal function, including motility, viability, fertilisation rates and initial cleavage rates can have damaged DNA that will result in reduced fertility and an increase in early pregnancy loss (Bathgate 2008). Changes in sperm chromatin structure and DNA integrity have been widely related to infertility in several mammalian species, and it is recommended that chromatin integrity should be studied as an independent complementary parameter for a better assessment of sperm quality. Sperm chromatin structure assays (SCSA), comet assays and the sperm chromatic dispersion test are some of the techniques currently applicable to evaluate boar sperm DNA fragmentation (Fraser \& Strzezek, 2005; Hernandez et al., 2006; Lopez-Fernandez et al., 2008)

In recent decades, laboratory assessments of either fresh or processed semen have evolved to become more detailed and have moved from the diagnosis of sperm attributes to determining the fertilising potential of the sample using in vitro tests such as zona pellucida (ZP) and oviductal epithelium binding assays, in vitro penetration assays or in vitro maturation/in vitro fertilisation/ in vitro production (IVM/IVF/IVP) systems (Rodriguez-Martinez 2003).

In 2005, Waberski et al. reviewed the procedures developed to evaluate the functional competence of boar spermatozoa by analyzing in vitro sperm-oocyte and sperm-oviduct interactions. In the former case, the ability of spermatozoa to interact with the ZP is evaluated by means of a sperm-zona binding assay, a sperm-zona penetration assay or an in vitro fertilisation assay, whereas in the latter case, the main aim is to assess multiple functions of the sperm plasma membrane that must be carried out before fertilisation.

Experiments conducted in our laboratory demonstrate that the evaluation of penetration rates and the average number of spermatozoa per penetrated oocyte using zona intact pig oocytes at the germinal vesicle stage (immature oocytes) in a homologous in vitro penetration (hIVP) assay provides a useful way to accurately predict male fertility (Martínez et al. 1993, 
1996). This assay is simple and rapid as it avoids the in vitro maturation of oocytes. Other parameters such as pronuclear formation and early embryonic development can be obtained using IVM/IVF/IVP systems. However, the problems of polyspermic penetration of oocytes and the poor quality of the embryo culture medium remain in the porcine IVF system, resulting in low-efficiency production of viable embryos and making their use for the prediction of fertility difficult (Gil et al. 2005).

The sperm-oviductal epithelium binding assay is emerging as an important tool for evaluation of sperm functionality since it evaluates plasma membrane quality for the multiple functions necessary prior to the fertilisation process (Waberski et al. 2005). Since binding to the oviductal epithelium stabilises the spermatozoa and enhances their survival in a hostile environment, it is clear that the ability of sperm cells to bind to the oviduct is a very important attribute intimately related to fertilising ability (Petrunkina et al. 2007). Along this line, several studies performed using this assay have demonstrated that the binding index was found to be lower in sperm samples coming from sub-fertile sires compared to that of fertile sires (Petrunkina et al. 2007, Waberski et al. 2005).

\section{How sperm technologies affect boar sperm fertilising ability}

Artificial insemination (AI) could be considered the most relevant sperm technology used worldwide in the reproductive management of domestic mammals (Weitze 2000). The manipulation of boar semen for Al generally involves two main steps consisting of dilution and, in most cases, storage of extended spermatozoa. Although this protocol is less stressful for the spermatozoa, some detrimental effects on their fertilising ability may be induced, especially if long storage periods are required (Vazquez et al. 1998, de Ambrogi et al. 2005).

The situation is different when sophisticated sperm technologies are applied to the boar gametes. Special characteristics of the boar sperm plasma membrane composition determine the extreme sensitivity of spermatozoa to cooling and freeze-thaw processes as well as to procedures that involve high dilution rates such as sex sorting by flow cytometry (de Graaf et al. 2008, Vazquez et al. 2009). This peculiarity of the male pig gamete is likely one of the most important limiting factors in the commercial use of these technologies for swine production.

Cryopreservation of spermatozoa involves several steps (temperature reduction, cellular dehydration, freezing and thawing) that may compromise sperm viability and normal function (Medeiros et al. 2002). Detrimental effects of the cryopreservation procedure include damage to the sperm plasma membrane and other organelles as a result of osmotic stress, cold shock and intracellular ice formation (Guthrie \& Welch 2005).

Over the years, great efforts have been dedicated to reducing the negative effects of freezing procedures on boar spermatozoa by introducing several improvements in sperm cryopreservation protocols (Eriksson et al. 2001, Carvajal et al. 2004, Saravia et al. 2005). As result of these investigations, sperm survival rates above $50 \%$ have been achieved along with promising fertility results (Roca et al. 2006).

Despite the improvements and the hopeful results, post-thaw viability, lifespan and fertility of cryopreserved boar spermatozoa are still reduced as a consequence of cellular injuries that arise during cryopreservation procedures (Medeiros et al. 2002) and remain at sub-optimal levels (Sancho et al. 2007). These facts together with the great variability in sperm freezability among boars and the large number of spermatozoa required per Al dose has discouraged the extensive use of frozen semen in commercial swine production (Johnson et al. 2000).

As noted above, the short lifespan of boar spermatozoa following cooling and freeze-thaw processes is one of the major drawbacks to the successful application of cryopreserved boar 
spermatozoa in commercial Al programmes, and it explains the low fertility results obtained (Roca et al. 2006). Premature capacitation (Watson 2000) and/or premature ageing (Peña et al. 2009) suffered by post-thaw spermatozoa have been pointed out as the main cause for the reduction in sperm lifespan, and thereby in fertilising capability. In either case, the reduction has been related to alterations in the cholesterol content of sperm membranes as well as with excessive formation of reactive oxygen species (ROS) as a consequence of the cryopreservation technique (Roca et al. 2004).

As occurs in cryopreservation procedures, flow cytometric sorting of $\mathrm{X}$ - and $\mathrm{Y}$-chromosome bearing boar spermatozoa involves several steps that can induce stress, damage or even kill spermatozoa. These steps include DNA staining, exposure to the UV-laser beam, high working

pressures, high dilution rates in different extenders (which may involve changes in osmolarity and $\mathrm{pH}$ ), centrifugation to concentrate the cells and in some cases the further stress of the cryopreservation procedure itself (Bathgate, 2008, Vazquez et al. 2008a, Garcia et al. 2007). Both the physical effect of the sorting procedure and the high dilution rate cause alterations in the functionality of sorted boar spermatozoa (Parrilla et al. 2005). However, the extensive dilution of spermatozoa (from approximately $800 \times 10^{6}$ in a sperm-rich fraction to a final concentration of sorted sperm of around $1 \times 10^{6}$ according to Bathgate, 2008) has been identified as the main determinant factor in the reduction of sorted sperm functionality. This fact might be due to the removal of seminal plasma factors required for the maintenance of sperm plasma membrane integrity and sperm functionality (Maxwell \& Johnson, 1999, Centurion et al. 2003, Caballero et al. 2004, 2008).

Therefore, as a result of all the manipulations needed for sperm sorting by flow cytometry, a stressed cell population with destabilised plasma membranes and showing capacitationlike changes is obtained (Maxwell \& Johnson 1999). This status renders the spermatozoa immediately able to fertilise an oocyte whether under in vivo or in vitro conditions without the need to perform further capacitation treatments (de Graaf et al. 2008). Detrimental effects caused by this premature capacitation status include reduced viability, lower fertilising ability and compromised storage capability after sorting (Maxwell \& Johnson 1999, Parrilla et al. 2005, see Figs 2 and 3). From a practical point of view, the reduction in the lifespan of sorted boar spermatozoa is an important drawback for the application of sexed semen in pig farming because, in most cases, great distances must be travelled between sperm-sorting laboratories and Al facilities (Vazquez et al. 2009).

Another important disadvantage of sorting technology when applied to $\mathrm{Al}$ in pigs is the reduction in fertility parameters with regard to farrowing rates and litter size (Vazquez et al. 2003, Garner 2006, Johnson et al. 2005). Whether this alteration in the reproductive parameters is a consequence of the low number of sorted sperm used - which may be unable to produce the minimum number of embryos necessary to maintain the pregnancy - or is due to alterations in sorted sperm DNA - resulting in the poor developmental potential of embryos - remains unclear. Further investigations into this phenomenon are clearly necessary (Bathgate 2008, Vazquez et al. 2009).

\section{Strategies for optimising the characteristics of spermatozoa subjected to technological treatments}

The handling of spermatozoa for sperm cryopreservation and sperm sexing by flow cytometry usually includes the concentration and the dilution of the spermatozoa, processes that produce a detrimental effect on the sperm cells. Consequently, new approaches are needed to improve these steps. 

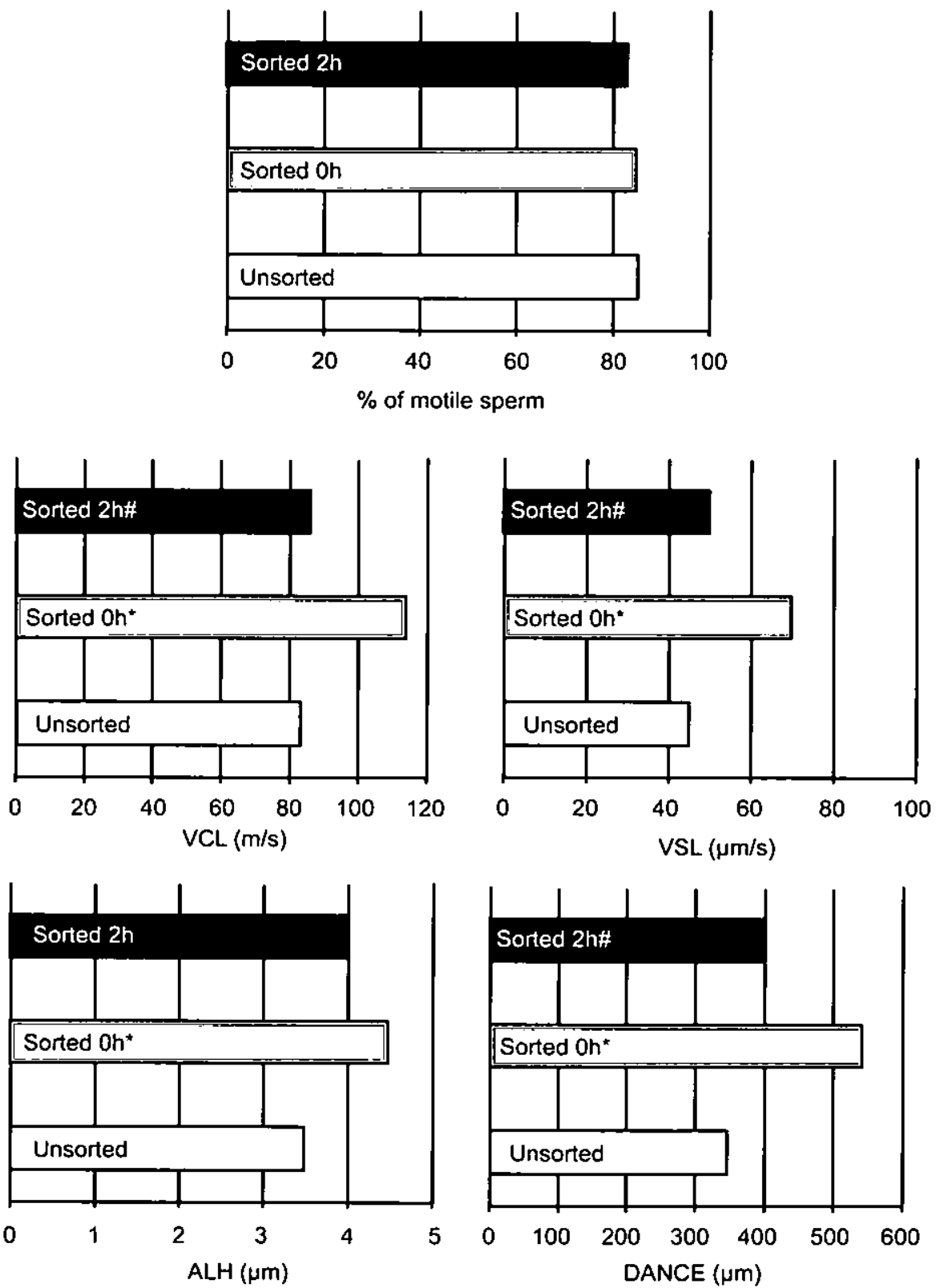

Fig.2 Changes in motion parameters in unsorted and sorted spermatozoa analyzed by CASA at 0 (sorted $\mathrm{Oh}$ ) and 2 hours (sorted $2 \mathrm{~h}$ ) of storage after sorting in the presence of seminal plasma. The percentage of motile sperm was maintained at around $80 \%$ up to $2 \mathrm{~h}$ of storage. Among the other variables explored, sperm velocity (VCL or VSL) and angularity parameters (AL.H and Dance) increased significantly $(p<0.05)$ immediately after sorting (sorted $\mathrm{Oh}$ ) only to return to unsorted sperm values after $2 \mathrm{~h}$ of storage in the presence of seminal plasma. VCL: Curvilinear velocity; VSL: Straight-line velocity; ALH: Amplitude of lateral head displacement; Dance: Curvilinear velocity multiplied by amplitude of lateral head displacement. ${ }^{*} \mathrm{P}<0.05$ compared to the unsorted sample, \# $\mathrm{P}<0.05$ compared to sorted Oh (modified from Parrilla et al., 2005). 
A

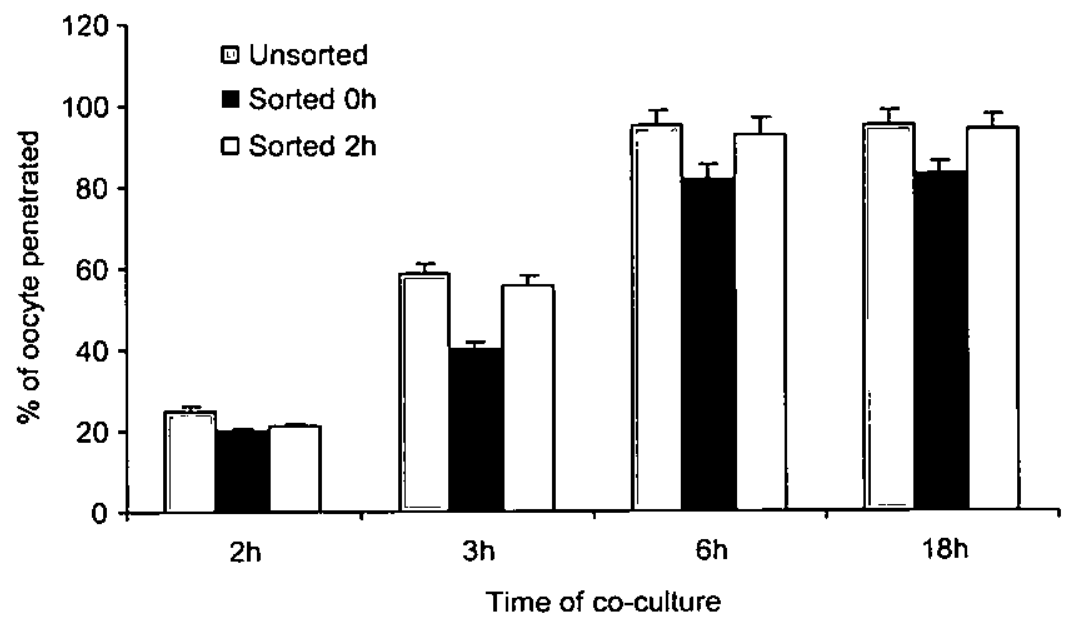

B

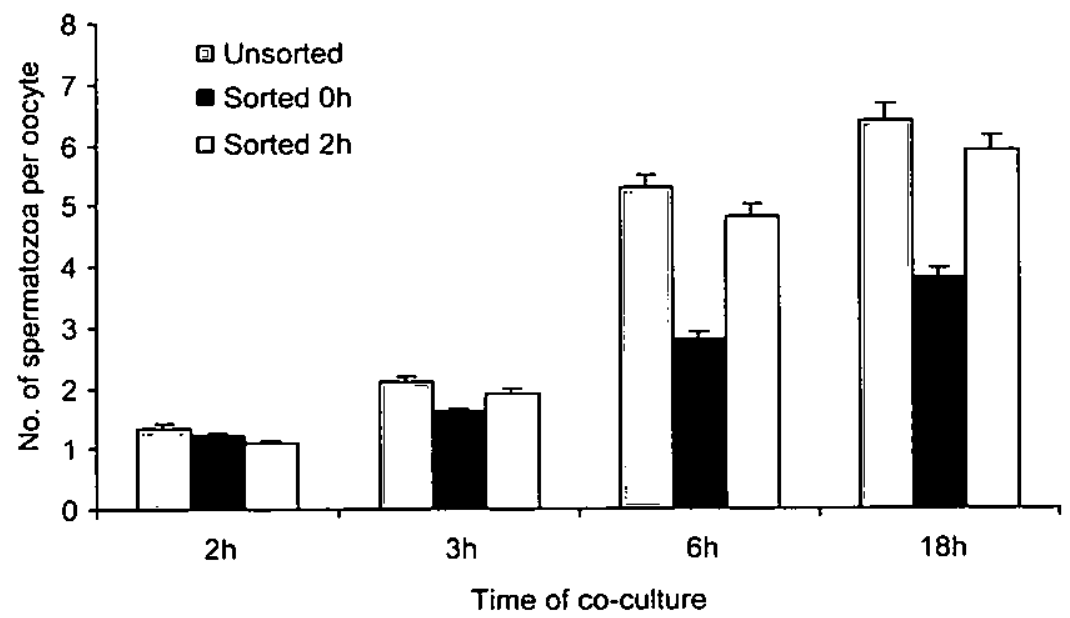

Fig. 3 Effects of flow sorting and flow sorting and storing in the presence of seminal plasma (SP) on time-course $(2,3,6,18$ hours) penetration rates (A) and the number of spermatozoa per oocyte (B). Sorted $\mathrm{Oh}$ : Spermatozoa sorted and co-incubated just after sorting; Sorted $2 \mathrm{~h}$ : Spermatozoa sorted and stored for $2 \mathrm{~h}$ in presence of SP. The histogram shows how spermatozoa sorted and stored for $2 \mathrm{~h}$ in the presence of SP had higher penetration rates and number of sperm per oocyte. This difference may be because the flow sorting procedure induces a scrambling in the plasma membrane components, affecting glycoproteins related to oocyte recognition and penetration. Incubation with SP for $2 \mathrm{~h}$ might reorganize the plasma membrane, restore the fertilizing capability and increasing the percentage of penetrating spermatozoa. ${ }^{*}$ indicates significant differences $(p<0.05)$ between histogram bars (modified from Parrilla et al., 2005).

\section{Methodologies for sperm concentration}

Although centrifugation is the usual procedure used for concentration of spermatozoa, it has been described as a process with detrimental effects on sperm cell membranes, reducing its 
fertilising capacity (Katkov \& Mazur 1998, Carvajal et al. 2004). Moreover, the induced damage appears to be worse when centrifugation is applied to "weak" spermatozoa, such as those that have been sex-sorted (Garcia et al. 2007). Sedimentation, as an alternative to centrifugation, can be use to concentrate boar spermatozoa, improving the in vivo fertilising ability of sex sorted boar spermatozoa (Garcia et al. 2007).

\section{Implementation of additives to the sperm extenders}

Improving the sperm environment through the use of an optimal medium is a principal strategy to overcome the low sperm quality obtained after application of sperm technologies. Addition of seminal plasma, certain proteins from the seminal plasma and antioxidant substances to the media surrounding the male reproductive cell have been used with cryopreserved and sex-sorted boar sperm with promising results (Maxwell \& Johnson 1999, Roca et al. 2004, Centurión et al .2003, Garcia et al. 2007).

\section{Seminal plasma}

Seminal plasma (SP) is the biological fluid in which the spermatozoa are bathed. It is well known that SP contains factors that influence both the spermatozoa and the female genital tract (Mann \& Lutwak-Mann 1982, Rozeboom et al. 2000). Most sperm technologies include the removal or extreme dilution of SP, a process that has been associated with a decrease in sperm quality and the acquisition of capacitation-like changes that lead to a shortening of sperm lifespan (Maxwell \& Johnson 1999). Therefore, a common counter-measure to alleviate these detrimental effects has been the addition of a certain proportion of SP to sperm extenders (Maxwell \& Johnson, 1997). However, there is considerable variability in the results reported in the literature regarding the effect of SP on frozen-thawed spermatozoa (Caballero et al. 2004, Maxwell et al. 2007, de Graaf et al. 2008). Initial research in sperm cryopreservation showed that pre-freezing spermatozoa in their own SP protected against cold shock (Pursel et al. 1973). On the other hand, Kawano et al. (2004) showed an improvement in sperm cryosurvival when SP was removed immediately after collection. Similar results have been reported regarding the addition of seminal plasma to post-thaw extenders with either improved membrane status (Vadnais et al. 2005) or diminished viability of the cryopreserved spermatozoa (Ericksson et al. 2005).

The application of SP to sex-sorted spermatozoa has been more straightforward. The addition of a certain proportion of SP (usually $10 \%$ ) to the collection media of sex-sorted spermatozoa improves sperm viability (Maxwell \& Johnson 1999). This protective effect is associated with a decrease in the capacitation-like changes observed in the sex-sorted spermatozoa (Maxwell \& Johnson 1997, Parrilla et al. 2005. See Figs 2 and 3). Although the beneficial effect of SP on sex-sorted sperm is somewhat consistent, as demonstrated by its inclusion in the collection media of most modern boar sperm sorting protocols (Grossfeld et al. 2005), such an effect is, however, boar-dependent (Caballero et al. 2004). These differences are associated with the variability observed in the SP composition among males as well as between ejaculates or fractions of the same ejaculate (Caballero et al. 2004, Rodríguez-Martínez et al. 2005, 2008, Saravia et al. 2009). Furthermore, a similar donor-dependent effect has been observed regarding sperm freezability, where the addition of SP from boars with good freezability to freezing extenders improved sperm cryosurvival (Hernández et al. 2007). These differences are prompting researchers to study the effects of specific components of the SP, such as SP proteins. 
Seminal plasma proteins

In boars, the bulk of SP proteins ( $>90 \%$ ) belongs to the spermadhesin family, a group of 12-16 kDa glycoproteins that bind to the sperm surface. Depending on their binding capability, spermadhesins can be classified into heparin-binding (AQN-1, AQN-3, AWN) and non-heparinbinding spermadhesins (PSP-I/PSP-II heterodimer) (Töpfer-Petersen et al. 1998). The PSP-I/ PSP-II heterodimer exerts a protective effect on highly diluted spermatozoa (Centurión et al. 2003). Based on immunolocalisation studies, PSP-I/PSP-II heterodimer has been seen to bind to the acrosomal cap of the spermatozoa. The binding of the heterodimer to the sperm surface seems to stabilise the plasma membrane of the spermatozoa (Caballero et al. 2006), delaying capacitation-like events, such as the increase in intracellular calcium and remodelling of the surface proteins of the sperm membrane (Caballero et al. 2006, 2009). This protective effect points to the PSP-I/PSP-1l as a candidate additive for extending the viability of technologically treated spermatozoa. In this way, the PSP-1/PSP-11 heterodimer has been tested as an additive for sex-sorted spermatozoa, showing a similar protective effect as that observed with SP (García et al. 2007). Experiments carried out in our laboratory using laparoscopic insemination demonstrated that the fertilising ability of sex-sorted spermatozoa collected in the presence of the PSP-I/PSP-II heterodimer increased when the PSP-I/PSP-II heterodimer was combined with sedimentation (instead of centrifugation) to concentrate the sperm sample (García et al. 2007).

\section{Antioxidants}

Sperm technologies often include procedures that are associated with the generation of ROS, which are related to defective sperm function (Muiño-Blanco et al. 2008). The use of antioxidants has been proposed to diminish the generation of ROS. Addition of catalase and superoxide dismutase reduced post-thaw ROS generation, improving sperm motility, viability and the ability of frozen-thawed spermatozoa to produce embryos in vitro (Roca et al. 2005). A beneficial effect of antioxidants was also seen with regard to the quality of flow-cytometrically sorted frozenthawed bull spermatozoa (Klinc \& Rath 2007), suggesting that the addition of antioxidants could be complementary for improving the fertilising ability of sex-sorted spermatozoa. Unfortunately, there are no references regarding the effects of antioxidant addition to the media used in sex sorting protocols for boar sperm.

\section{Other strategies to optimise sperm treatment technology outputs}

Application of sperm cryopreservation or sperm sex sorting in pigs generally implies the use of a small or very small number of weak spermatozoa. Together with the particular characteristics of the anatomy and reproductive physiology of the sow, this fact should be taken into account for the development and optimisation of protocols that allow the achievement of optimal fertility results after in vivo fertilisation.

Different strategies have been proposed in recent years to obtain optimal fertility results from insemination with a reduced number of boar sperm. These new procedures are mainly related to the site of semen deposition. In an attempt to deposit the spermatozoa as close as possible to the location of fertilisation by post-cervical insemination (intrauterine insemination, Watson \& Behan 2002), deep intrauterine insemination (Martinez et al. 2001) and laparoscopic intraoviductal insemination (García et al. 2007, Vazquez et al. 2008a, 2008b) procedures have been described and successfully used in combination with cryopreserved (Roca et al. 2003) and sex-sorted spermatozoa (Vazquez et al. 2003). In the case of sex-sorted spermatozoa, it 
has also been demonstrated that, in addition to the insemination procedure, the effect of the time of insemination relative to ovulation has an important influence on the success of the insemination procedure (reviewed by Vazquez et al. 2008a, 2008b).

\section{Concluding remarks}

Although it is clear that optimal sperm characteristics are those that allow the spermatozoa to fertilise an oocyte with subsequent embryo development, better knowledge of the characteristics of spermatozoa subjected to different technologies and an accurate prediction of fertilising ability by the application of the most appropriate sperm assessment assays are essential for optimising the yields of these techniques.

Reduced viability, lifespan and low fertility after the application of boar spermatozoa subjected to different sperm technologies have been widely described by several authors. These are the main drawbacks to overcome before these technologies are ready for application at commercial level. Important achievements have been made in cryopreservation and sex sorting protocols by modifying sperm handling procedures as well as through the addition of different substances. The consequent improvements in sperm functionality have been described by several different laboratories.

Furthermore, it should not be forgotten that fertilisation is a process that not only depends on sperm factors but also largely affected by aspects of the Al procedure and the physiology and management of the female. Al methods that allow deposition of the spermatozoa as close as possible to the site of ovulation and also as close to the ovulation time as possible are currently the most powerful tools for optimising the in vivo fertility of boar spermatozoa that have been subjected to cryopreservation or flow sorting.

As a general conclusion, it should be noted that important progress has been made in sperm technologies and many promising results have been achieved, which will be helpful for introducing these technologies on to pig farms. However, further research efforts should be pursued in order to facilitate a fully successful introduction of these sperm technologies to the swine production industry.

\section{Acknowledgements}

The authors are grateful to A. Fazeli for helpful suggestions and for critically reading the manuscript. Financial support from CICYT (AGF2005-00760, AGL2008-04127) and SENECA (04543/GERM07).

\section{References}

Amann RP, Hammerstedt RH \& Veeramachaneni DN 1993 The epididymis and sperm maturation: a perspective. Reproduction Fertility \& Development $5361-381$

Bathgate R 2008 Functional integrity of sex-sorted, frozen-thawed boar sperm and its potential for artificial insemination. Theriogenology 70 1234-1241.

Bussalleu E, Pinart E, Yeste M, Briz M, Sancho S, GarciaGil N, Badia E, Bassols J, Pruneda A, Casas I \& Bonet S 2005 Development of a protocol for multiple staining with fluorochromes to assess the functional status of boar spermatoazoa. Microscopy Research and Technique 68 277-283.

Caballero I, Vazquez JM, Rodríguez-Martinez $\mathbf{H}$, Roca J, Calvete JJ, Sanz L, Centurion F, Parrilla I \& Martinez EA 2004 Comparative effects of autologous and homologous seminal plasma on the viability of largely extended boar spermatozoa. Reproduction in Domestic Animals 39 370- 375.

Caballero I, Vazquez JM, Garcia EM, Roca J, Martinez EA, Calvete J, Sanz L, Ekwall \&, Rodriguez-Martinez H $2006 \mathrm{Imm}$ unolocalization and possible functional role 
of PSP-I/PSP-II heterodimer in highly extended boar spermatozoa. Journal of Andrology 27 766-773.

Caballero I, Vazquez IM, Mayor GM, Almiñana C, Calvete IJ, Sanz L, Roca / \& Martinez EA 2009 PSP-1/ PSP-11 spermadhesin exert a decapacitation effect on highly extended boar spermatozoa. International Journal of Andrology 9. (In press) Doi: 10.1111/j.13652605.2008.00887.x

Caballero I, Vazquez JM, García EM, Parrilla I, Roca J, Calvete Jl, Sanz L \& Martínez EA 2008 Major proteins of boar seminal plasma as a tool for biotechnological preservation of spermatozoa. Theriogenology $\mathbf{7 0}$ 1352-1355

Carvajal G, Cuello C, Ruiz M, Vazquez IM, Martinez EA \& Roca / 2004 Effects of centrifugation before freezing on boar sperm cryosurvival. Journal of Andrology 25 389-396.

Centurión F, Vazquez JM, Calvete J, Roca J, Sanz L, Parrilla I, Garcia EM \& Martinez EA 2003 Inf́luence of porcine spermadhesins on the susceptibility of boar spermatozoa to high dilution. Biology of Reproduction $69640-646$.

Cremades T, Roca J, Rodriguez-Martinez H, Abaigar T, Vazquez JM \& Martinez EA 2005 Kinematic changes during the cryopreservation of boar spermatozoa. Journal of Andrology 26 610-618

De Ambrogi M, Ballester \}, Saravia F, Caballero I, Anders J, Wallgren M, Magnus A, Andersson M \& RodriguezMartinez H 2005 Effect of storage in short- and longterm commercial semen extenders on the motility, plasma membrane and chromatin integrity of boar spermatozoa. International journal of Andrology 29 543-552.

de Graff SP, Leahy T, Marti J, Evans \& Maxwell WMC 2008 Application of seminal plasma in sex-sorting and sperm cryopreservation. Theriogenology 70 1360-1363.

Didion BA 2008 Computer-assisted semen analysis and its utility for profiling boar semen samples. Theriogenology 70 1374-1376.

Eriksson BM, Vazquez JM, Martinez EA, Roca J, Lucas X \& Rodriguez-Martinez H 2001 Effects of holding time during cooling and of type of package on plasma membrane integrity, motility and in vitro oocyte penetration ability of frozen-thawed boar spermatozoa. Theriogenology 55 1593-1605.

Eriksson BM, Bathgate R, Maxwell WMC \& Evans G 2005 Effect of seminal plasma protein fractions on boar spermatozoa motility and acrosome integrity. Theriogenology 63491.

Fraser L \& Strzezek / 2005 Effects of freezing-thawing on DNA integrity of boar spermatozoa assessed by the neutral comet assay. Reproduction in Domestic Animals 40 530-536.

Garcia EM, Vazquez JM, Parrilla I, Calvete J, Sanz L, Caballero I, Roca J, Vazquez JL \& Martinez. EA 2007 Improving the fertilizing ability of sex sorted boar spermatozoa. Theriogenology 68 771-778.

Garner DL 2006 Flow cytometric sexing of mammalian sperm. Theriogenology 65 943-957.
Gil MA, Roca J, Cremades $T$, Hernández $M$, Vázquez JM, Rodriguez-Martínez H \& Martinez EA 2005 Does multivariate analysis of post-thaw sperm characteristics accurately estimate in vitro fertility of boar individual ejaculates?. Theriogenology 64 305-316.

Graham JK \& Mocé E 2005 Fertility evaluation of frozen/ thawed semen. Theriogenology 64 492-504.

Grossfeld R, Klinc P, Sieg B \& Rath D 2005 Production of piglets with sexed semen employing a nonsurgical insemination technique. Theriogenology 63 2269-2277.

Guthrie HD \& Welch GR 2005 Effects of hypothermic liquid storage and cryopreservation on basal and induced plasma membrane phospholipid disorder and acrosome exocytosis in boar spermatozoa. Reproduction Fertility \& Development 17 467-477.

Guthrie HD \& Welch GR 2006 Determination of intracellular reactive oxygen species and high mitochondrial membrane potential in Percoll-treated viable boar sperm using fluorescence-activated flow cytometry. Animal Science 84 2089-2100.

Hallap T, Nagy S, Jaakma U, Johannisson A \& RodriguezMartinez $H 2005$ Mitochondrial activity of frozenthawed spermatozoa assessed by MitoTracker Deep Red 633. Theriogenology 63 2311-2322.

Harrison RAP \& Gadella BM 2005 Bicarbonate-induced membrane processing in sperm capacitation. Theriogenology 63 342-351.

Hernandez M, Roca J, Ballester J, Vazquez JM, Martinez EA, Johannisson A, Saravia F \& Rodriguez-Martinez H 2006 Differences in SCSA outcome among boars with different sperm freezability. International journal of Andrology 29 583-591.

Hernández M, Roca J, Calvete J, Sanz L, Muiño-Blanco T, Cebrián-Pérez JA, Vázquez JM \& Martínez EA 2007 Cryosurvival and in vilro fertilizing capacity postthaw is improved when boar spermatozoa are frozen in the presence of seminal plasma from good freezer boars. journal of Andrology 28 689-697.

Holt WV \& Van Look KJ 2004 Concepts in sperm heterogeneity, sperm selection and sperm competition as biological foundations for laboratory tests of semen quality. Reproduction 127 527-535.

Holt WV, O'Brien I \& Abaigar T 2007 Application and interpretation of computer-assissted sperm analyses and sperm sorting methods in assisted breeding and comparative research. Reproduction fertility \& Development 19 709-718.

Johnson L.A, Guthrie HD, Fiser P, Maxwell WMC, Welch GR \& Garret WM 2000 Cryopreservation of flow cytometrically sorted boar sperm: effects on in vivo embryo development. Journal of Animal Science 78 98 (abstract).

Johnson LA, Rath D, Vázquez JM, Maxwell WMC \& Dobrinsky IR 2005 Pre-selection of sex in swine for production of offspring: an update on the process and application. Theriogenology 63 615-624.

Katkov II \& Mazur P 1998 Influence of centrifugation regimes on motility, yield and cell associations of mouse spermatozoa. Journal of Andrology 19 
232-241.

Kawano N, Shimada M \& Terada T 2004 Motility and penetration competence of frozen-thawed miniature pig spermatozoa are substantially altered by exposure to seminal plasma before freezing. Theriogenology $61351-364$.

Klinc P \& Rath D 2007 Reduction of oxidative stress in bovine spermatozoa during flow cytometric sorting. Reproduction in Domestic Animals 42 63-67.

Lopez-Fernandez C, Perez-Llano B, García-Casado P, Sala R, Gosalbez A, Arroyo F, Fernandez JL \& Gosalvez J 2008 Sperm DNA fragmentation in a random sample of the Spanish boar livestock. Animal Reproduction Science 103 87-89.

Mann T \& Lutwak-Mann C 1982 Passage of chemicals into human and animal semen: mechanisms and significance. Critical Reviews in Toxicology 11 1.14.

Martinez E, Vazquez JM, Matas C, Roca J, Coy P \& Gadea f 1993 Evaluating of boar spermatozoa penetrating capacity using pig oocytes at the germinal vesicle stage. Theriogenology 40 547-557.

Martinez EA, Vazquez JM, Matas C, Gadea 1, Alonso MI \& Roca J 1996 Oocyte penetration by fresh or stored diluted boar spermatozoa before and after in vitro capacitation treatments. Biology of Reproduction 55 134-140.

Martinez EA, Vazquez JM, Roca J, Lucas X, Gil MA, Parrilla I, Vazquez JL \& Day BN 2001 Successful non-surgical deep intrauterine insemination with small numbers of spermatozoa in sows. Reproduction 122 289-96.

Martinez EA, Vazquez JM, Roca J, Cuello C, Gil MA, Parrilla I \& Vazquez IL 2005 An update on reproductive technologies with potential short-term application in pig production. Reproduction in Domestic Animals 40 300-309.

Maxwell WMC \& Johnson LA 1997 Chlortetracycline analysis of boar spermatozoa after incubation, flow cytometric sorting, cooling or cryopreservation. Molecular Reproduction and Development $\mathbf{4 6}$ 408-418.

Maxwell WM, Long CR, Johnson LA, Dobrinsky IR \& Welch GR 1998 The relationship between membrane status and fertility of boar spermatozoa after flow cytometric sorting in the presence or absence of seminal plasma. Reproduction Fertility \& Development 10 433-440.

Maxwell WMC \& Johnson LA 1999 Physiology of Spermatozoa at high dilution rates: the influence of seminal plasma. Theriogenology 52 1353-1362.

Maxwell WM, de Graaf SP, Ghaoui Rel-H \& Evans G 2007 Seminal plasma effects on sperm handling and female fertility. Society for Reproduction and Fertility Suppl. 64 13-38.

Medeiros CMO, Forell F, Oliveira ATD \& Rodrigues JL 2002 Current status of sperm cryopreservation: Why isn't it better?. Theriogenology 57 327-344.

Moran JM, Madejón L, Ortega Ferrusola C \& Peña FJ 2008 Nitric oxide induces caspase activity in boar spermatozoa. Theriogenology 70 91-96.

Muiño-Blanco T, Pérez-Pé R \& Cebrián-Pérez JA 2008 Seminal plasma proteins and sperm resistance to stress. Reproduction in Domestic Animals 43 18-31.

Parrilla I, Vazquez IM, Gil MA, Caballero I, Almiñana C, Roca J \& Martinez EA 2005 Influence of storage time on functional capacity of flow cytometrically sex-sorted boar spermatozoa. Theriogenology $6486-98$.

Peña Fl, Johannisson A, Wallgren M \& RodríguezMartínez H 2003 Assessment of fresh and frozenthawed boar semen using an Annexin- $V$ assay: a new method of evaluating sperm membrane integrity. Theriogenology 60 677-689.

Peña FJ, Saravia F, Johannisson A, Walgren M \& Rodríguez-Martínez H 2005 A new and simple method to evaluate early membrane changes in frozenthawed boar spermatozoa. International fournal of Andrology 28 107-114.

Peña FJ, Saravia F, Johannisson A, Wallgren W \& Rodriguez-Martínez H 2007 Detection of early changes in sperm membrane integrity pre-freezing can estimate post-thaw quality of boar spermatozoa. Animal Reproduction Science 97 74-83

Peña Fl, Rodriguez-Martinez H, Tapia \}, Ortega-ferrusola C, Gonzalez Fernandez L \& Macias Garcia B 2009 Mitochondria in mammalian sperm physiology and pathology: A review. Reproduction in Domestic Animal 44 345-349.

Petrunkina AM, Volker G, Brandt H, Töpfer-Petersen E \& Waberski D 2005 Functional significance of responsiveness to capacitating conditions in boar spermatozoa. Theriogenology 64 1766-1782.

Petrunkina AM, Waberski D, Günzel-Apel AR \& TöpferPetersen E 2007 Determinants of sperm quality and fertility in domestic species. Reproduction 134 3-17.

Piehler E, Petrunkina AM, Ekhlasi-Hundrieser M \& Töpfer-Petersen E 2006 Dynamic quantification of the tyrosine phosphorylation of the sperm surface proteins during capacitation. Cytometry A. 69 1062-70.

Pursel VG, Johnson LA \& Schulman LL 1973 Effect of dilution, seminal plasma and incubation period on cold shock susceptibility of boar spermatozoa. Journal of Animal Science 37 528-531.

Roca J, Carvajal G, Lucas X, Vazquez IM \& Martinez EA 2003 Fertility of weaned sows after deep intrauterine insemination with a reduced number of frozen-thawed spermatozoa. Theriogenology $6077-87$.

Roca J, Gil MA, Hernandez M, Parrilla I, Vazquez IM \& Martinez EA 2004 Survival and Fertility of boar spermatozoa after freeze-thawing in extender supplemented with butylated hydroxytoluene. Journal of Andrology 25 397-405.

Roca J, Rodriguez-Martinez H, Vazquez JM, Bolarin A, Hernandez $M$, Saravia $F$, Wallgren $M$ \& Martinez EA 2005 Strategies to improve the fertility of frozenthawed boar semen for artificial insemination. In Control of Pig Reproduction VIt, pp 261-275. Eds C) Ashworth and Kraeling RR, Nottingham University Press, Nottingham.

Roca J, Vazquez JM, Gil MA, Cuello C, Parrilla I 
\& Martinez EA 2006 Challenges in pig artificial insemination. Reproduction in Domestic Animals 41 43-53.

Rodriguez-Martínez H 2003 Laboratory semen assessment and prediction of fertility: still utopia? Reproduction in Domestic Animals 38 312-318.

Rodriguez-Martinez $H$, Saravia F, Wallgren $M$, Tienthai $P$, Johannisson A, Vázquez JM, Martínez $E$, Roca J, Sanz L \& Calvete II 2005 Boar spermatozoa in the oviduct. Theriogenology $63514-535$

Rodriguez-Martinez H 2007 State of the art in far animal sperm evaluation. Reproduction Fertility \& Development 19 91-101.

Rodríguez-Martínez $\mathrm{H}$, Saravia F, Wallgren $\mathbf{M}$, Roca J \& Peña FJ 2008 Influence of seminal plasma on the kinematics of boar spermatozoa during freezing. Theriogenology 70 1242-1250

Rozeboom KI, Troedsson MH, Hodson HH, Shurson GC \& Crabo BG 2000 The importance of seminal plasma on the fertility of subsequent artificial inseminations in swine. Journal of Animal Science 78 443-448

Sancho S, Casas I, Ekwall H, Saravia F, RodriguezMartinez $H$, Rodriguez-Gil JE, Flores E, Pinart E, Briz M, Garcia-Gil N, Bassols J, Pruneda A, Bussalleu E, Yeste M \& Bonet S 2007 Effects of cryopreservation on semen quality and the expression of sperm membrane hexose transporters in the spermatozoa of Iberian pigs. Reproduction $134111-121$.

Saravia F, Wallgren M, Nagy S, Johannisson A \& RodríguezMartínez H 2005 Deep freezing of concentrated boar semen for intra-uterine insemination: effects on sperm viability. Theriogenology 63 1320-1333.

Saravia F, Núñez-Martínez 1, Morán JM, Soler C, Muriel A, Rodriguez-Martínez H \& Peña FJ 2007 Differences in boar sperm head shape and dimensions recorded by computer-assisted sperm morphometry are not related to chromatin integrity. Theriogenology 68 196-203.

Saravia F, Wallgren M, Johannisson A, Calvete J, Sanz L, Peña FJ, Roca J \& Rodriguez-Martínez H 2009 Exposure to the seminal plasma of different portions of the boar ejaculate modulates the survival of spermatozoa cryopreserved in MiniFlatPacks. Theriogenology $71662-675$.

Silva PF \& Gadella BM 2006 Detection of damage in mammalian sperm cells. Theriogenology 65 958-978.
Töpfer-Petersen E, Romero A, Varela PF, EkhlasiHundrieser M, Dostàlovà Z, Sanz L. \& Calvete IJ 1998 Spermadhesins: a new protein family. Facts, hypotheses and perspectives. Andrologia 30 217-224.

Vadnais ML, Kirkwood RN, Specher DJ \& Chou K 2005 Effects of extender, incubation temperature, and added seminal plasma on capacitation of cryopreserved, thawed boar sperm as determined by chlortetracycline staining. Animal Reproduction Science 90 347-354.

Vazquez IM, Martinez EA, Roca J, Matas C \& Blanco O 1998 The fertilizing ability assessment of fresh and stored boar semen. Reproduction in Domestic Animals 33 267-270.

Vazquez JM, MartinezEA, Parrilla I, Roca J, Gil MA \& Vazquez JL 2003 Birth of piglets after deep intrauterine insemination with flow cytometrically sorted boar spermatozoa. Theriogenology 59 1509-1614.

Vazquez IM, Parrilla I, Gil MA, Cuello C, Caballero I, Vazquez JL, Roca J \& Martínez EA 2008a Improving the efficiency of insemination with sex-sorted spermatozoa. Reproduction in Domestic Animals. $431-8$.

Vazquez JM, Parrilla I, Gil MA, Cuello C, Caballero I, Vazquez JL, Roca J \& Martinez EA 2008b Low-Dose Insemination in Pigs: Problems and Possibilities. Reproduction in Domestic Animals 43 347-354.

Vazquez JM, Parrilla I, Roca J, Gil MA, Cuello C, Vazquez IL \& Martínez EA 2009 Sex-sorting sperm by flow cytometry in pigs: Issues and perspectives. Theriogenology 71 80-88.

Waberski D, Magnus F, Mendoca Ferreira F, Petrunkina AM, Weitze KF \& Töpfer-Petersen E 2005 Importance of sperm-binding assay for fertility prognosis of porcine spermatozoa. Theriogenology 63 470-484.

Watson PF 2000 The causes of reduced fertility with cryopreserved semen. Animal Reproduction Science 60-61 481-492.

Watson PF \& Beham IR 2002 Intrauterine insemination of sows with reduced sperm numbers: results of a commercially based field trial. Theriogenology 57 1683-1693.

Weitze KF 2000 Update on the worldwide application of swine AI. In Boar Semen Preservation N, pp 141-146. Eds Johnson LA, Guthrie HD. Allen Press 